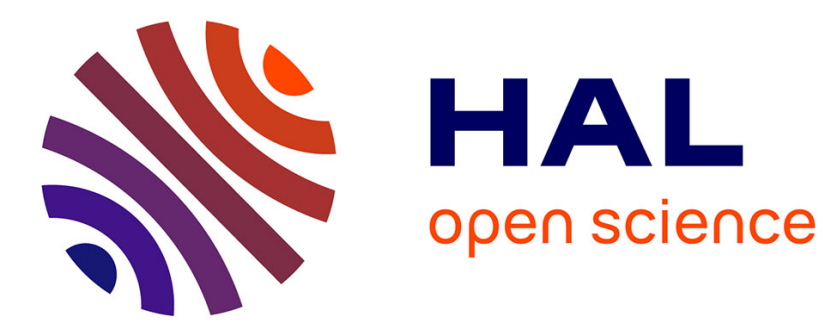

\title{
Potentiels démographiques pour des alternatives dans les campagnes françaises
}

Pierre Pistre

\section{To cite this version:}

Pierre Pistre. Potentiels démographiques pour des alternatives dans les campagnes françaises. Mouvements : des idées et des luttes, 2015, 84, pp 48-55. 10.3917/mouv.084.0048 . hal-01245101v2

\section{HAL Id: hal-01245101 \\ https://hal.science/hal-01245101v2}

Submitted on 23 Oct 2017

HAL is a multi-disciplinary open access archive for the deposit and dissemination of scientific research documents, whether they are published or not. The documents may come from teaching and research institutions in France or abroad, or from public or private research centers.
L'archive ouverte pluridisciplinaire HAL, est destinée au dépôt et à la diffusion de documents scientifiques de niveau recherche, publiés ou non, émanant des établissements d'enseignement et de recherche français ou étrangers, des laboratoires publics ou privés. 
Version pre-print de l'article du même titre paru dans la revue Mouvements, $n^{\circ} 84$, pp. 48-55.

Potentiels démographiques pour des alternatives dans les campagnes françaises

\section{PAR PIERRE PISTRE*}

À partir de plusieurs sources de données publiques, dont le dernier recensement de la population, l'auteur souligne la diversité démographique et économique des campagnes françaises d'aujourd'hui. L'évolution de ces contextes spatiaux est souvent mal connue ou caricaturée. Le constat d'une croissance de la population dans une majorité de territoires de campagne, même isolés, du fait de l'installation de nouveaux habitants, tranche avec l'idée répandue de " désertification démographique ». Ces dynamiques, pour partie, sont liées aux projets alternatifs qui se développent aujourd'hui dans ces territoires.

En septembre 2014, le journaliste indépendant Éric Dupin publiait Les défricheurs, voyage dans la France qui innove autrement'. Il y dépeignait l' « univers bigarré d'une autre France », composée de «nouveaux dissidents», d' écomilitants», d'«alterentrepreneurs », de «nouveaux paysans », à la recherche d'autres voies que le modèle productiviste et consumériste. Implicitement, il mettait aussi en évidence le poids des campagnes comme espaces privilégiés de développement d'alternatives variées : «Certains départements sont très riches en la matière, comme l'Ardèche, la Drôme, la Loire-Atlantique ou le Gers ${ }^{2} »$.

Les caractéristiques contemporaines de ces espaces en recomposition restent mal connues du plus grande nombre ou souvent caricaturées. Par ailleurs, l'analyse précise de la réalité des campagnes françaises et de leurs transformations actuelles peut conduire à s'interroger sur les potentiels et les freins à un développement plus significatif

* Géographe, post-doctorant à l'IFSTTAR et chercheur associé à l'UMR8504-Géographie-cités.

1 É. Dupin, Les défricheurs, voyage dans la France qui innove autrement, Paris, Éditions La Découverte, 2014.

2 Ibid., p. 11. 
des alternatives qui y émergent. Cet article s'emploie à éclairer ces contextes spatiaux, par une perspective d'étude nationale à dominante quantitative. Les éléments de synthèse présentés dans l'article découlent de l'exploitation de différentes sources de données publiques récentes (recensement de la population 2007 et $2012^{3}$, impôt sur le revenu des personnes physiques 2011, démographie des établissements économiques 2009 et 2013, base permanente des équipements 2011 et 2014). Des références sont aussi faites à d'autres publications personnelles, qui font suite à un travail de thèse de géographie sur le thème du renouveau démographique des campagnes françaises ${ }^{4}$.

\section{Pluralité des campagnes et outil d'analyse de leur diversité}

Nous définissons les campagnes par opposition aux villes denses et nombreuses. Qu'est-ce qui les caractérise aujourd'hui ? Le maintien de l'activité agricole ? La faible densité de population ? Le poids des espaces naturels ? Un peu tout cela mais, à l'échelle nationale, elles forment surtout une mosaïque d'espaces, eux-mêmes divers dans leurs compositions (pluriactivité, populations résidentes, etc.). Le temps des campagnes paysannes est ainsi révolu et la place de l'agriculture y est déclinante sauf exception. Les densités et les espaces artificialisés y restent plus faibles qu'ailleurs, mais les croissances de population sont aussi de plus en plus généralisées et les paysages loin d'être figés.

Plusieurs chercheurs ont produit récemment des classifications statistiques du territoire français pour analyser les campagnes dans leur diversité, à distance du point de vue urbano-centré qui les uniformise comme un hors-ville. Même si l'influence urbaine, surtout métropolitaine, est grandissante, il s'agit en premier lieu d'adopter une définition extensive des campagnes qui intègre dans le spectre de l'analyse une majorité d'espaces dits périurbains, dont les moteurs d'évolution sont loin d'être exclusivement urbains.

La grille d'analyse ici mobilisée est la «typologie des campagnes françaises », produite par un groupe de chercheurs de l'Inra, du laboratoire ThéMa et de l'Irstea, pour

3 Les données de recensement sont exactement collectées sur 5 ans (ex : RP 2012, de 2010 à 2014).

4 Voir P. PISTRE, Renouveaux des campagnes françaises : évolutions démographiques, dynamiques spatiales et recompositions sociales, Thèse de doctorat, Université Paris Diderot, 2012, https://halshs.archives-ouvertes.fr/tel-00764869/ 
le compte de la Datar. Cet outil d'analyse prend en compte les communes qui n'appartiennent pas aux zones agglomérées, par continuité du bâti, de plus de 10000 emplois. Elles sont classées en fonction de nombreux indicateurs statistiques qui correspondent à trois entrées thématiques : les populations et leurs conditions de vie (accessibilité, mobilité), les dynamiques économiques (marché de l'emploi, secteurs d'activités) et le cadre paysager (occupation du sol, relief). La définition plurielle des campagnes qui en résulte repose ainsi sur les caractéristiques intrinsèques et variées des communes. Cette approche plurifactorielle diffère en particulier du zonage en aires urbaines produit par l'Insee. En dehors des pôles urbains, les espaces y sont en effet catégorisés comme «périurbains », «multipolarisés » ou « isolés hors de l'influence des pôles » selon les seuls trajets domicile-travail des actifs (à savoir si plus ou moins $40 \%$ des navettes se font vers une ou plusieurs aires urbaines).

Sous cet angle d'étude, les campagnes représentent aujourd'hui environ $44 \%$ de la population de la France métropolitaine pour $93 \%$ des communes. Trois grands types de campagnes sont distingués selon leurs composantes et leurs positions spatiales.

On trouve d'abord les campagnes des villes, du littoral et des vallées urbanisées, les plus peuplées (16,8 millions d'habitants), aux économies diversifiées et dynamiques (commerce, construction, etc.), qui enregistrent les plus fortes croissances de population (+ 766500 habitants entre 2007 et 2012). Les populations résidentes des premières couronnes des grandes agglomérations s'y démarquent notamment par des revenus élevés (29 800 euros en moyenne par foyer fiscal) - même supérieurs à ceux enregistrés dans les unités urbaines de plus de 10000 emplois (28 400 euros) - et une forte présence des actifs cadres (16\%) ou des professions intermédiaires $(28 \%)$.

On trouve ensuite les campagnes agricoles et industrielles, concentrées dans la moitié nord du pays, qui sont sous moindre influence urbaine. La densité de population y est plus faible (en moyenne 540 habitants par commune), les populations beaucoup moins diplômées et les revenus moyens intermédiaires. Surtout, l'agriculture en nombre d'emplois (13\%) et d'établissements économiques (35\%), d'une part, et l'industrie à travers la forte présence d'ouvriers (31\% des actifs résidents), d'autre

5 Voir M. Hilal, A. BarczaK, F.-P. TourneuX, Y. Schaeffer, M. Houdart, E. CRemerSCHULTE, «Typologie des campagnes françaises et des espaces à enjeux spécifiques », Travaux en 
part, y restent les principaux marqueurs spatiaux.

Dernier type principal, les campagnes vieillies à très faible densité. Réparties sur l'ensemble du territoire, elles ont en commun un degré supérieur de vieillissement de leur population (environ autant d'habitants de 65 ans et plus que de moins de 24 ans), un peuplement épars et de (très) faibles revenus moyens par foyer fiscal (plus de $50 \%$ de foyers fiscaux non imposables). Les plus étendues du nord-est au sud-ouest - certainement la figure la plus classique des campagnes françaises dans l'imaginaire collectif — restent marquées par le poids de l'agriculture (environ $25 \%$ des emplois pour $50 \%$ des établissements économiques) et la présence importante des ouvriers (28\% des actifs résidents). Les campagnes à dominante de montagnes (Alpes, Pyrénées, Corse, Sud du Massif Central) se distinguent par contre par une économie tournée vers les services, aussi bien de l'administration publique (11 \% des emplois) que de l'hôtellerie et de la restauration (11\% également).

\section{Les campagnes dans les grandes tendances de la société}

Si les campagnes contemporaines ont de multiples visages, elles n'en sont pas moins inscrites dans de nombreuses tendances de société qui concernent l'ensemble du territoire. Analyser leurs transformations récentes passe donc en premier lieu par l'examen de la matérialisation de ces dynamiques d'évolution bien plus générales. Elles sont à la fois sociodémographiques et socioéconomiques, qu'il s'agisse du vieillissement de la population, de la perte de vitesse des secteurs industriels et agricoles, ou encore de la tertiarisation des professions et des activités économiques.

Le vieillissement de la population prend par exemple la forme d'un accroissement généralisé de la part des populations d'âge intermédiaire, surtout des jeunes retraités ( $c f$. générations du baby-boom), et des populations les plus âgées ( $c f$. amélioration de l'espérance de vie). En effet, si les structures en âge des populations restent inégales dans l'espace, la tendance au vieillissement apparaît assez comparable entre les campagnes (ex : de $+1,1$ à $+1,3$ points de croissance des 40-64 ans entre 2007 et 2012) - ce surplus étant pour partie lié aux dynamiques migratoires ( $c f$. partie suivante). Le vieillissement de la population se matérialise aussi par un développement significatif

ligne, 12, Paris, Datar, 2012, https://halshs.archives-ouvertes.fr/hal-00911232/. 
du secteur de l'hébergement médicosocial et de l'action sociale, à destination première des personnes âgées, qui a le plus progressé en proportion des emplois dans les campagnes comme dans les villes (de +1 à $+1,7$ points entre 2007 et 2012).

Soulignons ensuite le moindre poids des ouvriers, surtout non qualifiés, qui découle plus généralement du déclin de l'industrie dans le marché de l'emploi. Espaces de campagnes et espaces urbains sont tous concernés, mais en premier lieu les régions d'ancienne spécialisation industrielle du nord du pays (- 2,2 points des actifs résidents entre 2007 et 2012 dans les campagnes agricoles et industrielles). Pour autant, la part des ouvriers dans la société reste élevée, et en particulier dans les campagnes ${ }^{6}$ où ils représentent souvent encore la première catégorie professionnelle des résidents actifs. Les dynamiques migratoires montrent aussi une surreprésentation des ouvriers parmi les nouveaux résidents du périurbain lointain ou des campagnes les moins denses?

La perte de vitesse du secteur agricole se poursuit par ailleurs dans l'ensemble des configurations de campagnes, en tout cas dans une perspective d'analyse nationale. Sur le long terme, la tendance est plutôt au tassement de la décroissance des effectifs d'emplois et d'établissements économiques liés à l'agriculture. Néanmoins, sur la seule période 2007-2012, elle reste le premier secteur en perdition dans les différentes campagnes, en particulier dans les campagnes vieillies de très faible densité (- $20500 \mathrm{em}$ plois de l'agriculture, de la sylviculture et de la pêche, soit $8 \%$ des effectifs de 2007).

Les campagnes contemporaines sont enfin pleinement inscrites dans la tendance générale à la tertiarisation des professions et des activités économiques. Elle prend par exemple la forme d'une croissance significative des actifs résidents des professions de services, des secteurs privé et public, en particulier des professions intermédiaires (de $+1,3$ à $+1,7$ points entre 2007 et 2012 selon les campagnes). La progression est structurelle, du fait de l'évolution du marché de l'emploi et de l'élévation du niveau de formation des populations; elle est aussi migratoire, au regard de la représentation de ces populations parmi les nouveaux résidents de tout ou partie des campagnes.

\section{Tableau 1 - Évolution récente de la couverture de certains services et équipements}

6 Julian Mischi a notamment décrit le poids encore important des ouvriers dans les territoires ruraux. Voir «Les territoires ruraux, des espaces ouvriers en mutation », Métropolitiques, 2013 (en ligne) et l'article publié dans ce numéro. 


\begin{tabular}{|c|c|c|c|c|}
\hline \multirow[b]{2}{*}{$\begin{array}{l}\text { Variation du taux de couver- } \\
\text { ture entre } 2011 \text { et } 2014 \\
\text { (situations au } 1 \text { er janvier) }\end{array}$} & \multicolumn{4}{|c|}{ Typologie des campagnes françaises } \\
\hline & $\begin{array}{c}\text { Campagnes } \\
\text { des villes, } \\
\text { du littoral } \\
\text { et des } \\
\text { vallées } \\
\text { urbanisées }\end{array}$ & $\begin{array}{l}\text { Campagnes } \\
\text { agricoles et } \\
\text { industrielles }\end{array}$ & $\begin{array}{l}\text { Campagnes } \\
\text { vieillies à } \\
\text { très faible } \\
\text { densité }\end{array}$ & $\begin{array}{c}\text { Unités } \\
\text { Urbaines } \\
\text { supérieures } \\
\text { à } 10000 \\
\text { emplois }\end{array}$ \\
\hline \multicolumn{5}{|c|}{ [1] Services aux particuliers, [2] Commerce, [3] Enseignement, [4] Santé } \\
\hline [1] Gendarmerie & $-{ }_{-}^{-}$ & 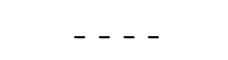 & -- & (cf. police) \\
\hline [1] Banque & - & -- & --- & + \\
\hline [1] Restaurant & ++ & + & + & + \\
\hline [2] Hyper-/Supermarché & +++ & +++ & +++ & +++ \\
\hline [2] Supérette et épicerie & - & -- & - & +++ \\
\hline [2] Boulangerie & - & - & - & + \\
\hline [3] École maternelle & -- & --- & --- & -- \\
\hline [3] École élémentaire & -- & - & - & - \\
\hline [3] Collège & -- & -- & -- & -- \\
\hline $\begin{array}{l}\text { [4] Etablissement de santé } \\
\text { pour courts à longs séjours }\end{array}$ & - & -- & -- & ---- \\
\hline [4] Médecin omnipraticien & - & - & - & - \\
\hline [4] Infirmier & ++ & +++ & ++ & +++ \\
\hline
\end{tabular}

Évolution du taux de couverture moyen d'un équipement/service entre 2011 et 2014 (en nombre d'habitants par équipement/service) :

Dégradation $=-$ [0 à 50 habitants] ; - - 50 à 100 habitants $]$; - - - [100 à 500 habitants] ; - - - - [500 à 1000 habitants] ; - - - - - [Supérieur à 1000 habitants]

Amélioration $=+[0$ à 50 habitants $] ;++[50$ à 100 habitants $] ;+++[100$ à 500 habitants $]$; ++++ [500 à 1000 habitants] ; +++++ [Supérieur à 1000 habs]

Lecture : le taux de couverture correspond au nombre moyen d'habitants pour un équipement ou service selon chaque catégorie spatiale. L'évolution tient compte ainsi à la fois de la variation du nombre d'équipement ou service et du volume de la population résidente. On parle de dégradation (ou inversement d'amélioration) lorsque moins d'habitants sont en moyenne couvert par un équipement ou service. La fermeture ou la création d'équipements/services parmi les plus rares (hypermarché, hôpital, etc.) a mécaniquement tendance à augmenter fortement la dégradation/amélioration de la couverture.

Source : Insee, base permanente des équipements 2011 et 2014 ; Insee, recensement de la population 2009 et 2012

La tertiarisation se matérialise également par la croissance d'activités variées de services dans les économies locales. Sur ce point, l'analyse de la répartition des équipements et des services dans l'espace révèle par exemple la hausse de la couverture en restaurants, en hypermarchés/supermarchés ou en infirmiers, et ce même dans les campagnes vieillies à très faible densité (tableau 1). Toutefois, la période 2011-2014 a

7 Voir : P. PISTRE, op.cit., 2012. 
surtout été marquée par la forte dégradation de l'accès à de nombreux autres services et équipements : des services aux particuliers (ex : gendarmerie), certains commerces (ex : supérette, épicerie), les services de l'enseignement (ex : collège) ou de la santé (ex : médecin omnipraticien). Les services publics sont notamment concernés et, même si les politiques de restriction budgétaire ont touché l'ensemble des territoires, le ressentiment local a été encore plus fort dans bon nombre de campagnes. En effet, la dégradation récente est souvent venue aggraver des taux de couverture par habitant déjà parmi les plus mauvais, la faible densité spatiale impliquant en plus des distances supérieures à parcourir pour accéder à tel ou tel service public.

\section{Gains de population grâce aux dynamiques migratoires}

Si nombre des tendances d'évolution des campagnes relèvent de dynamiques sociétales plus générales, il est un domaine où elles divergent du reste du territoire : celui des leviers d'évolution des populations résidentes. L'image d'espaces en perdition démographique, surtout par « exode migratoire », reste bien ancrée dans les mentalités et dans certains discours médiatiques. Pour autant, l'analyse chiffrée nationale renvoie une autre représentation. Bien sûr, beaucoup de communes des campagnes continuent de perdre de la population - elles sont même un peu plus nombreuses depuis la fin des années 2000, après la crise économique de 2008-2009 - , mais la majorité sont aujourd'hui en croissance, et ce, même dans les campagnes vieillies à très faible densité.

À l'inverse des gains de population enregistrés dans les unités urbaines, les croissances dans les campagnes résultent surtout de l'amélioration de leur solde migratoire (différence entre arrivées et départs). Dans les années 1960-1970, seules les campagnes des villes et les zones touristiques accueillaient plus de nouveaux habitants qu'elles n’enregistraient de départs. Depuis les années 1980, la hausse des installations et la diminution des sorties concernent les différentes configurations de campagnes, jusqu'à atteindre des soldes positifs comparables entre 2007 et 2012 (taux de croissance annuel moyen de la population dû au solde migratoire de $+0,4$ à $+0,8 \%)$. Par contre, les évo-

8 Pour des détails chiffrés sur les dynamiques démographiques et migratoires dans les espaces les moins denses, voir P. PISTRE, «Recensements de la population pour l'étude des reprises démographiques et des migrations résidentielles dans l'espace peu dense (1975-2011)», Économie et statistique, 2015 (à paraître). 
lutions naturelles (naissances et décès) restent très différenciées, et les soldes négatifs dans la plupart des campagnes les moins denses.

Qui s'installe donc aujourd'hui dans les campagnes, notamment hors de l'influence urbaine directe ? À la périphérie des villes, les profils moteurs des développements périurbains des années 1970-1980 restent les plus représentés (jeunes couples avec ou sans enfants, classes moyennes : professions intermédiaires, employés, etc.), mais la stratification sociale des campagnes des villes s'est aussi nettement renforcée en fonction de la proximité aux (grandes) agglomérations.

Dans les espaces les moins denses, la tendance est à la diversification du profil des nouveaux résidents. Les (jeunes) retraités restent les plus représentés : déjà nombreux à s'y installer dans les années 1980, leur poids s'est affirmé de manière régulière depuis. Mais les installations des années 2000 ont été aussi le fait d'une diversité d'actifs en milieu et fin d'activité professionnelle. Il y a là des ouvriers, des employés, des artisans, des cadres, des professions intermédiaires, dont les migrations ont été guidées par des désirs de changement, des expériences passées, des contraintes... Une particularité est ici que de mêmes territoires locaux peuvent attirer des populations de tous niveaux sociaux, en raison notamment de leurs structures de logement très hétérogènes : par exemple, des situations de repli économique dans du locatif dégradé, peu cher, comme d'acquisition de grands bâtiments agricoles propices à la réalisation de biens d'exception.

J'ai pour partie étudié ces motivations variées au cours de ma thèse, à travers trois enquêtes de terrain menées en Haute-Provence, dans le Haut-Languedoc et au sudouest de la Haute-Vienne. Trois ensembles de motivations non exclusives sont revenus de manière récurrente dans les entretiens semi-directifs réalisées avec des observateurs locaux (élus, associatifs, acteurs de l'immobilier, etc.) et de nouveaux résidents (retraités, actifs) ${ }^{9}$. Le premier relève de la perception des lieux : tel ou tel territoire peu dense est choisi pour sa tranquillité, son cadre naturel, sa dimension humaine, par opposition aux maux de l'ancien lieu de résidence urbain (foisonnement, promiscuité, etc.). Le deuxième ensemble de démarches invite à replacer les derniers changements de

9 Pour plus de détail, voir P. PISTRE, «À la recherche d'un entre-deux spatial idéal, motivations d'installation dans les campagnes françaises », Carnets de géographes, 7, 2014 (en ligne). 
résidence dans les trajectoires personnelles plus longues. Que la migration ait été motivée par un retour aux «origines familiales» ou par des habitudes de vacances, le point commun est une pratique antérieure du nouveau lieu de résidence, et plus ou moins régulière au cours du cycle de vie.

Un dernier ensemble de motivations résidentielles est guidé par des formes de stratégies spatiales. Premier cas classique, conciliation est faite entre aspirations familiales à la vie à la campagne et nécessité professionnelle de la proximité urbaine. Le lieu d'implantation est alors largement fonction des prix de l'immobilier, mais au-delà d'une certaine distance, la capacité à assumer le surplus des coûts de la mobilité quotidienne devient le privilège de certains actifs des groupes moyens et supérieurs. Une autre forme de stratégie spatiale consiste à choisir des campagnes même assez isolées pour leur position d'entre-deux géographique. Il s'agit par exemple de couples d'actifs dont les lieux de travail sont localisés dans des villes distinctes. Il s'agit aussi de retraités qui s'installent dans des territoires en position intermédiaire - à l'échelle régionale voire nationale - par rapport aux lieux de résidence de leurs enfants et petits-enfants.

\section{Vers des campagnes alternatives ?}

Sauf à étudier des innovations ciblées et de plus en plus institutionnalisées ${ }^{10}$, l'étude générale des transformations des campagnes françaises laisse peu de place au monde des alternatives, alors même que les initiatives se multiplient. L'explication est pour partie méthodologique, du fait des outils statistiques utilisés. Mais les contextes spatiaux de campagne restent aussi marqués par des pesanteurs démographiques et économiques.

Pour autant, certaines des dynamiques récentes laissent entrevoir des potentiels d'alternatives économiques et sociales à travers les changements démographiques. Pour les années 1990, Yannick Sencébé et Denis Lepicier ont parlé d'« épiphénomène de la néoruralité » dans les migrations de l'urbain vers le rural, surtout isolé ${ }^{11}$. Sur le plan comptable, il est difficile d'affirmer que les installations récentes portées par des aspirations à des modes raisonnés de production agricole, à de nouvelles formes de gou-

10 Par exemple, les circuits courts agroalimentaires dont Chevallier et al. (2014) ont fait une évaluation précise en Limousin (cf. Géographie, économie, société, 16(3), p. 339-362).

11 Voir «Migrations résidentielles de l'urbain vers le rural en France : différenciation sociale des profils et ségrégation spatiale », EspacesTemps.net, 2007 (en ligne). 
vernance politique, ou encore à des logements alternatifs, ont été bien plus nombreuses que précédemment. Par contre, les motivations d'ensemble des nouveaux arrivants s'accompagnent le plus souvent d'aspirations à des modes de vie plus sains ou à un retour à des consommations locales, plus « écologiques » ou plus abordables. D'une manière générale, la diversification des populations des campagnes d'aujourd'hui, portée par les installations résidentielles, suggèrent que des économies équilibrées, résilientes, voire autonomes, pourraient émerger. Cette possibilité inverse ainsi l'idée d'une mise sous perfusion de ces marges spatiales par des économies métropolitaines globalisées.

Les perspectives d'alternatives diffèrent ensuite selon les configurations spatiales. Les campagnes des villes montrent par exemple un profil distinct des autres campagnes, parfois plus marqué encore que les villes elles-mêmes, notamment en termes de revenus. Elles bénéficient et dépendent du fonctionnement métropolitain en tant que couronnes périurbaines, leurs résidents actifs travaillant majoritairement dans les grands pôles urbains. Mais en même temps, elles constituent des îlots de pouvoir et de richesse qui tendent à repousser les ambitions intégratrices des pôles métropolitains. Sans vouloir renoncer au confort des services urbains, leurs populations sont aussi attachées à la préservation des traits campagnards de ces territoires d'entre-deux. Les enjeux d'innovation sociale paraissent précisément se situer ici dans la meilleure valorisation à faire de leur situation spatiale.

Autre cas au bout du spectre des campagnes, les territoires les plus vieillis et les moins denses semblent être l'image même du déclin rural et de la victoire de l'urbain par délaissement des territoires les plus isolés. Pourtant une majorité de ces espaces gagnent aussi des habitants par migration. Certains y voient la possibilité de réactiver une société paysanne, structurée par le travail de la terre, ou plus largement d'organiser un fonctionnement local fait d'autoproduction et de consommation sur place. Dans tous les cas, le défi des alternatives dans ces territoires peu denses résident aussi dans l'amélioration du quotidien des populations locales, pour lesquelles à la dispersion spatiale s'ajoute, plus souvent qu'ailleurs, la faiblesse des ressources économiques ${ }^{12}$.

12 Pour des précisions sur les précarités rurales actuelles, notamment dans les territoires les plus isolés, voir le numéro récent de la revue Pour sur le sujet, 225, 2015. 